\title{
Dose effects of mycophenolate mofetil in Chinese patients with neuromyelitis optica spectrum disorders: a case series study
}

\author{
Yujuan Jiao', Lei Cui', Weihe Zhang ${ }^{1}$, Chunyu Zhang ${ }^{2}$, Yeqiong Zhang ${ }^{1}$, Xin Zhang ${ }^{1}$ and Jinsong Jiao ${ }^{1 *}$ (D)
}

\begin{abstract}
Background: Neuromyelitis optica (NMO) spectrum disorder (NMOSD) is a devastating autoimmune inflammatory disorder of the central nervous system, which can result in blindness or paralysis. Currently, there is a dire need for new treatment options in the clinic. Several case series have shown that mycophenolate mofetil (MMF) may be an effective treatment for NMOSD patients. The dosing of MMF in the treatment of NMOSD has been poorly studied. Therefore, we evaluated the efficacy, tolerability, influential factors and optimal dosage of MMF in Chinese patients with NMOSD.
\end{abstract}

Methods: A case series of 109 NMO or NMOSD (limited forms of NMO with seropositive AQP4-lgG) patients were retrospectively analyzed and followed up. Out of the 109 patients, 86 patients had received MMF for 6 months or longer and were included for efficacy assessment.

Results: When comparing the annualized relapse rate (ARR) of MMF treatment with that of pre-MMF treatment period, MMF was found to significantly reduce ARR in $75(87 \%)$ patients $(p<0.0001)$. The median pre-treatment Expanded Disability Status Scale (EDSS) score in remission decreased from 3 (range, 0-8.5) to 2.5 (range, 0-8) at the last follow-up $(p=0.006)$, yet no significant difference was found in the visual score. The higher doses of MMF $(1750 \mathrm{mg} / \mathrm{d}$ to $2000 \mathrm{mg} / \mathrm{d})$ significantly lowered the relapse risks compared with lower doses $(1000 \mathrm{mg} / \mathrm{d}$ or less, $p<0.0001$ ) or moderate doses (1250 to $1500 \mathrm{mg} / \mathrm{d}, p=0.031$ ). Coexisting with systemic autoimmune diseases $(H R, 2.418 ; p=0.0345)$ and attack number before MMF initiation $(H R, 1.117 ; p=0.02)$ were important risk factors for relapses. MMF was generally well tolerated with adverse effects occurring in 21 patients (19\%). While four patients decreased their daily doses because of the adverse effects, only one patient stopped MMF treatment.

Conclusions: MMF is generally effective and well tolerated in Chinese NMOSD patients. High-dose MMF was more potent than the lower dose for NMOSD patients, with $1750 \mathrm{mg}$ of daily MMF being the recommended dosage for Chinese patients with NMOSD. MMF treatment reduces the frequency of relapses and improves the quality of life for patients with this debilitating disease.

Keywords: Mycophenolate mofetil, Neuromyelitis optica, Neuromyelitis optica spectrum disorders, Efficacy, Tolerability, Dose effects

\footnotetext{
* Correspondence: jiao_jinsong@163.com

1Department of Neurology, China-Japan Friendship Hospital, \#2 Yinghuayuan

East Street, Chaoyang District, Beijing 100029, China

Full list of author information is available at the end of the article
}

C The Author(s). 2018 Open Access This article is distributed under the terms of the Creative Commons Attribution 4.0 International License (http://creativecommons.org/licenses/by/4.0/), which permits unrestricted use, distribution, and reproduction in any medium, provided you give appropriate credit to the original author(s) and the source, provide a link to the Creative Commons license, and indicate if changes were made. The Creative Commons Public Domain Dedication waiver (http://creativecommons.org/publicdomain/zero/1.0/) applies to the data made available in this article, unless otherwise stated. 


\section{Background}

Neuromyelitis optica (NMO) spectrum disorder (NMOSD) is a devastating autoimmune inflammatory disorder of the central nervous system, which can lead to blindness or paralysis. The risk of developing disabilities increases significantly with the number of relapses [1,2]. Prevention of relapse is essential for the successful treatment of NMOSD patients. While there have been no placebo-controlled or comparative randomized controlled trials of immunosuppressive therapies conducted in NMO patients, several case series have reported that mycophenolate mofetil (MMF) may be effective for treatment of NMOSD [3-10]. To date, there are no clear recommendations regarding the dosing of MMF. In this study, we aimed to evaluate the efficacy, safety profile and recommendable dosage of MMF in a large cohort of Chinese patients with NMO and NMOSD.

\section{Methods \\ Patients}

This study was approved by the Medical Ethics Committee of China-Japan Friendship Hospital (2016-62). Patient consent forms were obtained from all patients or his/her legal representatives before the study. We performed a retrospective review of the medical records from patients that presented with NMO, using the 2006 revised NMO criteria [11] or the NMOSD (limited forms of NMO with seropositive AQP4-IgG) [12]. From January 2009 to October 2016, 109 patients (96 female and 13 males) received MMF treatment and were enrolled for individual tolerability assessments. Of the 109 patients, 86 received $\geq 6$ months of MMF were included for the efficacy assessment, 22 patients had recently initiated MMF treatment, and one patient stopped MMF before the end of 6 months due to an adverse reaction. Patients who received $1000 \mathrm{mg} / \mathrm{d}$ or less of MMF were classified as the low-dose treatment group, while MMF dosages of $1250 \mathrm{mg} / \mathrm{d}$ and $1500 \mathrm{mg} / \mathrm{d}$ were deemed as the moderate-dose treatment. The highest dosages utilized in this study were $1750 \mathrm{mg} / \mathrm{d}$ and $2000 \mathrm{mg} /$ $\mathrm{d}$, which were considered as the high-dose treatment. While receiving MMF treatment, each patient received long-term concomitant oral corticosteroids (10-15 mg every other day) for the first one to two years. At each follow-up appointment, routine blood tests were performed to assess the efficacy of the therapy. The patients were recommended to follow-up every 3 months, and there was a minimum annual follow-up requirement and all of the follow-up appointments were recorded.

\section{Clinical assessment}

Data was recorded for the patients, including demographic data, detailed treatment plans (daily dose of MMF and glucocorticoid, date and reason for the initiation or cessation of immunosuppressive agents, and the starting or stopping of any other treatments), clinical course, adverse reactions, modified Expanded Disability Status Scale (EDSS) and corrected visual acuity at remission and each follow-up appointment [1].

Visual acuity was assessed separately for each eye using the following scale: $0=20 / 20 ; 1=$ scotoma, but better than $20 / 30 ; 2=20 / 30$ to $20 / 59 ; 3=20 / 60$ to $20 / 199 ; 4=20 / 200$ to $20 / 800 ; 5=$ count fingers only; $6=$ light perception; $7=$ no light perception [1]. The visual outcome in remission was the sum of the visual scores for each eye after each attack and at the follow-up appointments.

A relapse is defined as a sudden worsening of neurological function lasting for more than $24 \mathrm{~h}$ that is unknown in origin with no other identifiable causes, such as a fever or infection. Additionally, a relapse will increase the EDSS score by a half point or more, or it may be indicated by a worsening of one point in two of the functional systems or two points in a single functional system. A severe relapse was defined as an EDSS score of six or more, which required a walking aid to travel $100 \mathrm{~m}$ with or without resting, at the nadir of the attack. In those patients with baseline EDSS scores $\geq 6.0$, an increase of 0.5 points or more was classified as a severe relapse. In cases of optic neuritis $(\mathrm{ON})$, a severe relapse was defined as a sudden worsening of visual acuity (VA) of 0.1 or less in patients with baseline VA scores of greater than 0.1. When accompanied with MRI evidence of ON, any decrease of VA was regarded as a severe relapse if the baseline vision was light perception, hand motion, or counting fingers [5, 9]. Suboptimal treatment with MMF was defined as 6 months or less of therapy or daily dosages less than the minimal therapeutic dose (1250 $\mathrm{mg}$ in adults).

\section{Statistical analyses}

Data were analyzed using SAS version 9.3 (SAS institution Inc., NC, USA). A two-sided $p \leq 0.05$ was considered statistically significant. The Wilcoxon signed-rank test was used to compare pre-treatment annualized relapse rates (ARR), EDSS, and visual scores with on-treatment indexes. The number of severe attacks that occurred before and during MMF treatment was compared using the Pearson chi-square test. Characteristics were compared among the different MMF dosage groups (i.e., female and male) using the Pearson chi-square test for categorical data and the Kruskal-Wallis $\mathrm{H}$-test for continuous data. The Kaplan-Meier method was used to determine the time to first relapse among different groups, and were then compared using the log-rank test. Hazard ratios (HR) that pertained to the first relapse after the start of MMF treatment were calculated using the Cox proportional hazard model, as follows:

$$
h(t, x)=h_{0}(t) \exp \left(\beta_{1} x_{1}+\beta_{2} x_{2}+\ldots+\beta_{m} x_{m}\right)
$$


where $t$ is the first relapse time, and $\mathrm{x}$ is the MMF dosage, concomitant with any systemic autoimmune diseases, preMMF ARR, pre-MMF EDSS, duration of MMF therapy, duration of pre-MMF, attacks number before MMF initiation, gender, age at onset and serum AQP4-IgG positivity.

\section{Results}

\section{Baseline demographic and clinical data}

The demographic and clinical characteristics of the cohort are summarized in Table 1. Diagnoses at the initiation of MMF therapy were NMO (64), transverse myelitis (9, recurrent in 7), recurrent optic neuritis (3), and NMOSD with other clinical characteristics (10). Among NMOSD patients receiving different dosages of MMF, there were no significant differences between the baseline

Table 1 Demographic characteristics of patients who received MMF treatment for six months or longer

\begin{tabular}{|c|c|}
\hline Characteristic & Value \\
\hline Number of Patients & $n=86$ \\
\hline Current age, median (range), y & $53(15-84)$ \\
\hline Female sex, No. (\%) & $77(90 \%)$ \\
\hline NMO diagnosis, No. (\%) & $64(74 \%)$ \\
\hline NMOSD diagnosis, No. (\%) & $22(26 \%)$ \\
\hline Aquaporin-4 antibody positivity, No. (\%) & $74(86 \%)$ \\
\hline Age at onset, median (range), y & $43(6-68)$ \\
\hline Overall disease duration, median (range), mo & $71(7-535)$ \\
\hline Disease duration before receiving MMF, median (range), mo & $71(6-444)$ \\
\hline Attack number before receiving MMF, median (range) & $5(1-33)$ \\
\hline Duration of MMF treatment, median (range), mo & $20(6-89)$ \\
\hline Abnormal autoantibodies ${ }^{a}, \mathrm{n}(\%)$ & $39(45 \%)$ \\
\hline Coexisting with systemic autoimmune diseases & $29(34 \%)$ \\
\hline Concurrent use of prednisone, $\mathrm{n}(\%)$ & $65(76 \%)$ \\
\hline treatment-naïve patients, $\mathrm{n}(\%)$ & $21(24 \%)$ \\
\hline \multicolumn{2}{|l|}{ Previous immunosuppressive agents: } \\
\hline Corticosteroids ${ }^{b}, \mathrm{n}(\%)$ & $33(38 \%)$ \\
\hline Azathioprine, n (\%) & $15(17 \%)$ \\
\hline Cyclophosphamide, n (\%) & $4(5 \%)$ \\
\hline Rituximab, n (\%) & $2(2 \%)$ \\
\hline Tacrolimus (FK506) & $1(1 \%)$ \\
\hline Methotrexate & $1(1 \%)$ \\
\hline \multicolumn{2}{|l|}{ Previous immunomodulatory therapies: } \\
\hline$\beta$-interferons, n (\%) & $6(7 \%)$ \\
\hline hydroxychloroquine sulfate, n (\%) & $2(2 \%)$ \\
\hline Mitoxantrones, n (\%) & $1(1 \%)$ \\
\hline
\end{tabular}

${ }^{a}$ Autoantibodies refers to rheumatoid factors, antinuclear antibodies, antidouble-stranded DNA antibodies, ribose nuclear proteins, anti-SM antibodies, anti-SSA and anti-SSB antibodies, TPO and TG antibodies

${ }^{b}$ Corticosteroids refers to continuously taking oral prednisone or methylprednisolone for more than 3 months characteristics including age, female percentage, complete NMO patient percentage, aquaporin-4 antibody positivity, age at disease onset, duration of MMF treatment, treatment-naïve patients, disease duration, attack number, ARR and EDSS before receiving MMF (Additional file 1: Table S1).

\section{Efficacy: MMF therapy significantly reduced ARR of NMOSD}

During a median course of 20 months (average 27) therapy with MMF, 55 (64\%) patients were relapse-free and 75 (87\%) of the 86 evaluated patients experienced improvement in their ARR. Among the 31 patients who relapsed during MMF therapy, 7 (23\%) patients experienced their first relapse within 6 months of initiating MMF therapy. The median ARR during MMF treatment (0, range 0-2.8) was significantly reduced $(p<0.0001$, Table 2$)$ compared with the pre-treatment ARR (1.4, range 0.1-11.0). The Kaplan-Meier survival estimated the significant difference between the relapse-free rates of pre-treatment and during treatment periods $(p<0.001$, Fig. 1a).

\section{Efficacy: MMF therapy significantly decreased the risk of severe relapses}

A total of 572 attacks were recorded in 86 patients with NMOSD. Of the 572 attacks, 502 (6 with uncertain severities) of them happened prior to the initiation of MMF therapy, including 200 (40\%) attacks rated as severe and 296 attacks rated as mild. During MMF treatment, only $16(23 \%)$ of the 70 relapses were severe. There was a significantly lower risk of patients experiencing severe relapses during MMF treatment when compared with the period prior to MMF therapy initiation $(p=0.006)$.

\section{Disability efficacy: MMF was effectual for improving disabilities in NMOSD}

The EDSS scores improved in 36 patients and were unchanged in 39 patients, which was 75 out of the 86 (87\%) NMOSD patients. There was a statistically significant decrease between the median EDSS score obtained at the beginning of MMF treatment (in remission) and at the last follow-up $(p=0.006$, Table 2$)$. The median visual scores obtained at pre-MMF treatment (in remission) and the last follow-up visit were 2 (average 3.0, range, $0-13$ ) and 1 (average 2.7, range, $0-13$ ), respectively. While the visual scores improved in 11 patients and stabilized in 68 patients (total of 79 out of 86 or 92\% NMOSD patients), there was no significant difference between values obtained at pre-treatment and the last follow-up $(P=0.106)$. 
Table 2 Subanalysis of treatment efficacy in patients treated with MMF

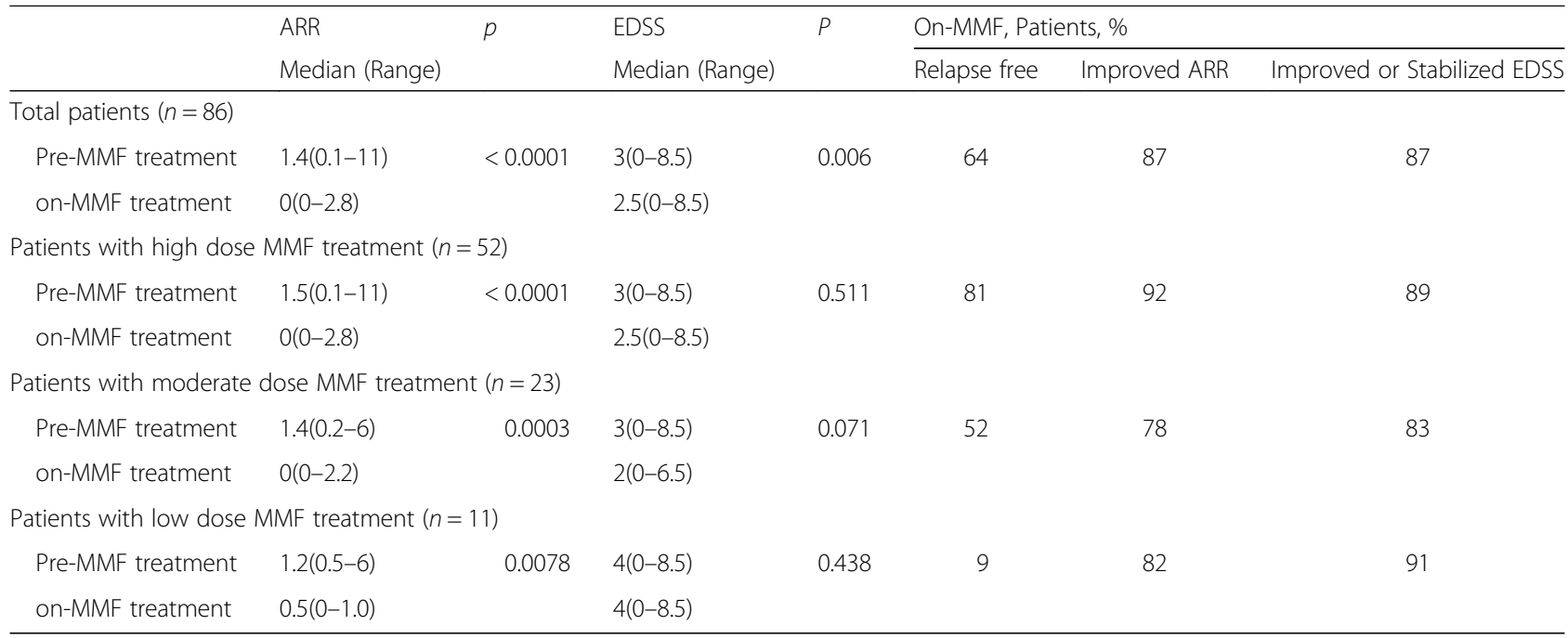

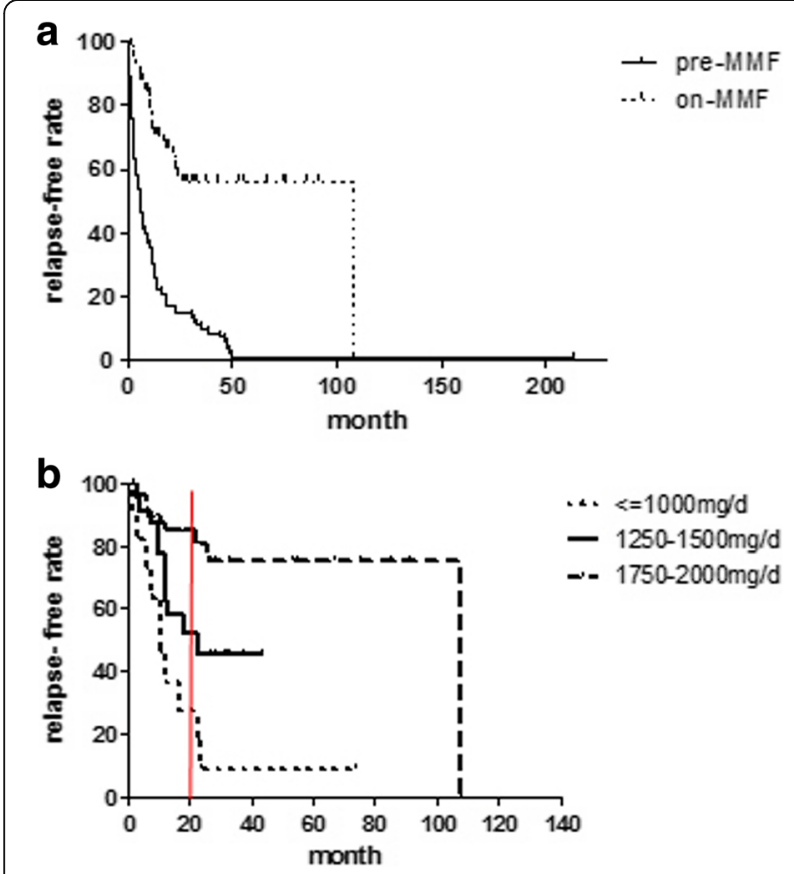

Fig. 1 Kaplan-Meier survival estimates pertaining to probabilities of being free of the occurrence of any relapse (a) between during MMF treatment (on-MMF, dashed line) and before MMF treatment initiation (pre-MMF, black line) in NMOSD patients (Log-rank test, $p<0.001$ ). The relapse-free rates after 1 year and 2 years therapy with MMF were $72 \%$ and $58 \%$, respectively. Those values were much higher than $30 \%$ and $14 \%$ before initiation of MMF therapy. (b) with different dose of MMF therapy. After 20 months of MMF therapy, approximately $68 \%$ and $42 \%$ of NMOSD patients in the high-dose and moderate-dose groups would remain relapse-free, respectively (Log-Rank test, $p=0.019$ ). However, only $9 \%$ of the patients receiving low-dose MMF would remain relapse-free, significant lower than the moderate or high-dose groups (Log-Rank test $p=0.031, p<0.0001$ )

\section{Efficacy: High dose MMF was more potent than lower dose for NMOSD}

Among 31 NMOSD patients who relapsed during MMF therapy, 10 of the 11 (91\%) patients were taking the lowest dosage of $1000 \mathrm{mg} / \mathrm{d}$ or less MMF relapsed, 11 of the 23 (48\%) patients were on the median dosage of $1250 \mathrm{mg} / \mathrm{d}$ or $1500 \mathrm{mg} / \mathrm{d}$, and 10 of the 52 patients (19\%) were receiving the highest dosage of $1750 \mathrm{mg} / \mathrm{d}$ or $2000 \mathrm{mg} / \mathrm{d}$ (Table 2). The proportion of patients on concomitant corticosteroids for more than 1 year and 2 years did not differ among the three doses. Statistically significant differences in relapsefree rates were found between the lower and moderate dosage groups $(p=0.031)$, moderate and higher dosage groups $(p=0.019)$, and the lower and higher dosage groups $(p<0.0001$, Fig. 1b). The adjusted hazard risks also indicated that the higher dosage of MMF was a protective factor for preventing relapse (Fig. 2). The EDSS scores were improved in 18 patients (35\%), 13 patients (57\%), and 5 patients $(46 \%)$ in the lower, moderate, and higher MMF dosage groups, respectively. Additionally, the EDSS scores remained unchanged in 28 patients (54\%), 6 patients (26\%), and 5 patients (46\%) in the lower, moderate, and higher MMF dosage groups, respectively. However, among the three MMF dosage groups, there is no statistically significant difference either in the number of patients with improved EDSS scores or in the number of patients with unchanged EDSS scores $(p=0.276)$.

\section{NMOSD patients coexist with concomitant systemic autoimmune diseases were more prone to relapses} There were 29 (34\%) NMOSD patients who had at least one systemic autoimmune disease in this study, which included 20 patients with thyroid disease, 7 patients with Sjögren syndrome, 4 patients with systemic lupus erythematosus, 2 patients with rheumatoid arthritis, 2 patients 


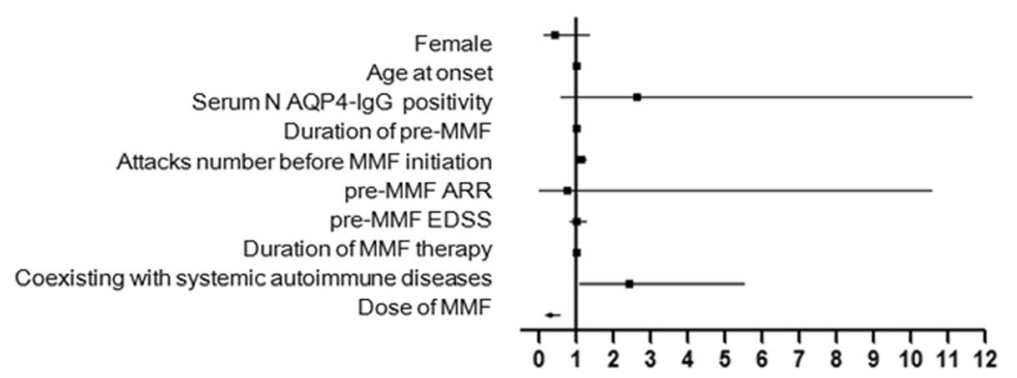

Fig. 2 Adjusted Hazard Risks for relapse after MMF initiation, according to the clinical characteristics and based on patients who received MMF for more than 6 months. NMOSD patients treated with the higher dose of MMF had a significantly lower risk of relapse (HR, 0.291; 95\% confidence interval, 0.164-0.516; $p<0.0001$ ). Significant risk factors for relapse in NMOSD patients were the presence of any systemic autoimmune disease (HR, 2.418; $95 \%$ confidence interval, $1.066-5.481 ; p=0.0345)$ and an increased number of relapses before MMF initiation (HR, 1.117; 95\% confidence interval, 1.018-1.227; $p=0.020$ ). Other factors were determined to have little influence on the effect of MMF treatment

with Castleman disease, 2 patients with psoriasis, 2 patients with interstitial pneumonia and 1 patient with myasthenia gravis. It was found that concomitant systemic autoimmune disease (HR, 2.418; 95\% confidence interval, 1.066-5.481; $p=0.0345$ ) and relapses numbers before the initiation of MMF treatment (HR, 1.117; 95\% confidence interval, 1.018-1.227; $p=0.02$ ) were significant risk factors for the relapse in NMOSD patients (Fig. 2).

\section{Side-effects and MMF tolerability}

Twenty-one of 109 patients (19\%) reported adverse effects with MMF treatment, including hair loss $(n=5)$, increased transaminase levels $(n=3)$, low white blood cell and neutrophil counts $(n=3$, one of these patients also reported interstitial pneumonia and another reported human papillomavirus type 1 [HPV-1] infection), diarrhea and abdominal pain $(n=2)$, shingles $(n=2)$, herpes simplex infection $(n=2)$, headache $(n=2)$, thrombocytopenia $(n=1)$, constipation $(n=1)$, and chronic dermopathy on the hands and nails $(\mathrm{n}=1)$. Five patients $(4.6 \%)$ reported moderate to severe adverse effects and among them, two patients treated with MMF $2000 \mathrm{mg} / \mathrm{d}$ were admitted to hospital due to increased transaminase levels and interstitial pneumonia, respectively. Of the two patients, one discontinued MMF in the first two months of treatment, while the dosage was decreased from $2000 \mathrm{mg} / \mathrm{d}$ to $1250 \mathrm{mg} / \mathrm{d}$ for the second patient. The dosage of three additional patients was lowered from $2000 \mathrm{mg} / \mathrm{d}$ to $1500 \mathrm{mg} / \mathrm{d}$ because of increased transaminase levels, HPV-1 infection, or low neutrophil counts, within six months of initiating MMF treatment. These side effects were mild and symptomatic treatment were effective.

\section{Discussion}

In this study, patients received MMF with concomitant low dose oral corticosteroids therapy in the first one to two years of MMF therapy. It was reported that the proportions of relapse-free patients that experienced improved ARR values and EDSS scores did not differ between patients treated with MMF alone and MMF in combination with prednisone [6]. Thus, we discuss the overall treatment of MMF with or without oral corticosteroids as MMF therapy. Similar to previous studies [3-10], MMF therapy significantly reduced ARR in $87 \%$ of patients, and $64 \%$ were relapse-free during a median course of 20 months (average 27) therapy with MMF. Our results confirmed that MMF therapy significantly decreased the risks of severe relapses, in terms of disability, as recently reported $[9,10,13]$.

For patients receiving MMF therapy, EDSS scores at last follow-up were improved and maintained in $87 \%$ of the NMOSD patients, which is similar to the percentage of patients that experiencing improvement in their ARR. Hence, MMF is an effective treatment for reducing disabling relapses in NMOSD. We compared the disability status in remission before or at the beginning of MMF treatment with those at the last follow-up. This was done to minimize the influence on EDSS scores reductions caused by corticosteroid impulse therapy and spontaneous recovery in the acute phase. It was reported that $68 \%$ to 97\% patients experienced improved or unchanged EDSS scores after MMF treatment $[4,9,12,14]$. This difference in results may be caused by the varying number of the patients in acute phase and treatment duration. In our study, the post-treatment visual scores, which were not mentioned in any of the previous studies, remained statistically unchanged, suggesting that visual deficiency is usually fixed and more difficult to improve than the disability caused by myelitis.

As of now, there are no standard criteria for determining the optimal dosage of MMF for treating NMOSD among treatment centers. As this is a retrospective study based on a review of medical records, the evaluated NMOSD patients were receiving different daily doses of MMF. While all dosage levels of MMF have shown some benefit in reducing relapse rates in NMOSD patients, the lower and moderate dosage carry a much higher risk of relapse. Nearly 9 out of 10 of NMOSD patients taking 
the lower dosage of MMF experienced a relapse, which was reduced to 5 out of 10 patients in the moderate dosage group. These results verified that $1500 \mathrm{mg} / \mathrm{d}$ or less MMF was insufficient for most of the Chinese NMOSD patients. While there were no significant differences in EDSS between the three different treatment groups, longer follow-up times could have revealed different results. Hence, longer follow-up or prospective controlled trials are necessary to validate these findings. Considering that nearly $20 \%$ of the patients receiving 1750 to $2000 \mathrm{mg} / \mathrm{d}$ of MMF still relapsed, natural history studies infer a stepwise accumulation of attack-related disability for most patients with NMOSD and any treatment failure is potentially devastating for NMOSD patients [2]. Overall, we found that high-dose MMF therapy provided the most benefit to NMOSD patients.

One-third of our NMOSD patients had at least one coexisting systemic autoimmune disease. Any coexisting systemic autoimmune disease was a significant risk factor for relapse. As another point of view, MMF was also effective for Sjögren syndrome, systemic lupus erythematosus, and several other autoimmune disorders [15-17], suggesting that high-dosage MMF may be an optimal treatment for NMOSD patients with coexisting systemic autoimmune diseases. While most of the moderate to severe adverse effects were reported by the patients receiving high-dosage MMF $(2000 \mathrm{mg} / \mathrm{d})$ therapy, pharmacokinetic studies may allow for individualization of MMF dosing for NMOSD patients in the future.

In this study, the number of relapses before the initiation of MMF was another risk factor for further relapses in NMOSD patients. This indicated that patients were more prone to relapse if they had several prior attacks. The usage, dosage, and timing of prophylactic agents are still being actively investigated for NMOSD patients. However, these results suggest an adequate immunosuppressant should be recommended for NMOSD patients with a history of relapse.

In terms of tolerability, MMF was generally well tolerated in the 109 Chinese NMOSD patients. Adverse effects were reported by 21 patients, and 5 of the 52 patients receiving high-dosage MMF $(2000 \mathrm{mg} / \mathrm{d})$ reported moderate to severe adverse effects that required therapeutic intervention. From this study and other case series and cohort studies published so far [3-10], MMF was administrated alone or in combination with oral corticosteroids in more than 500 NMOSD patients. No serious toxicity concerns, such as malignancies or progressive multifocal leukoencephalopathy, which had been reported in transplant patients when MMF was used in conjunction with other immunosuppressants [14].

The present study was limited by its absent blinding, retrospective design, and the uneven assignment of patients to the different dosage group. Specifically, more recently diagnosed patients were more likely to receive the higher dosage of MMF. Despite these methodological limitations, this study provides useful information on the influence factors and optimal MMF dosage choice for the treatment of NMOSD. In the future, randomized controlled trials will be necessary to further verify the finds from this study.

\section{Conclusion}

MMF is a safe and effective oral immunosuppressant for treatment of NMOSD. High-dosage MMF was more potent than the lower dose for treatment of NMOSD, with $1750 \mathrm{mg}$ of daily MMF being the recommended dosage for Chinese patients with NMOSD. As for NMOSD patients with coexisting systemic autoimmune diseases, a higher daily dosage of MMF may be recommended.

\section{Additional file}

Additional file 1: Table S1. Baseline demographic and clinical data of NMOSD patients receiving different dosages of MMF. There was no significant difference in baseline demographic and clinical data among the 3 groups of the NMOSD patients receiving different dosages of MMF. (DOC $41 \mathrm{~kb}$ )

\section{Abbreviations}

ALT: Alanine aminotransferase; AQP4: Aquaporin-4; ARR: Annualized relapse rate; AST: Aspartate aminotransferase; EDSS: Expanded Disability Status Scale; HPV: Human papillomavirus; HR: Hazard ratio; lgG: Immunoglobulin G; MMF: Mycophenolate mofetil; NMO: Neuromyelitis optica; NMOSD: Neuromyelitis optica spectrum of disorders; ON: Optic neuritis; VA: Visual acuity

\section{Acknowledgements}

We would like to thank laboratory technicians, Ying Hao and Jin Zhang, for collecting and processing the serum samples.

\section{Funding}

This work was supported by the Research Fund of the China-Japan Friendship Hospital (2013-RC-3) and the Foundation of Capital Characteristic Clinical Application Research (2106-BKJ-004).

\section{Availability of data and materials}

The data sets generated and analyzed during this study are available with the approval of the corresponding authors with reasonable requests.

\section{Authors' contributions}

JJ had full access to all the data in the study and takes responsibility for the integrity of the data and the accuracy of the data analysis. Study concept and design: YJ and JJ. Acquisition of data: YJ, LC, WZ, JJ, YZ and XZ. Analysis and interpretation of data: JJ, YJ, LC, WZ, CZ and XZ. Drafting of the manuscript: YJ. Critical revision of the manuscript for important intellectual content: All authors. Statistical analysis: YJ and CZ. Obtained funding: YJ and JJ. Supervised the study: JJ, YJ, LC and WZ. All authors read and approved the final manuscript.

\section{Ethics approval and consent to participate}

This study was approved by the Medical Ethics Committee of China-Japan Friendship Hospital. The reference number is: 2016-62. Written informed consents were obtained from all the participants or his/her parents/legal representatives (for participant under 18 years old).

Competing interests

The authors declare that they have no competing interests. 


\section{Publisher's Note}

Springer Nature remains neutral with regard to jurisdictional claims in published maps and institutional affiliations.

\section{Author details}

'Department of Neurology, China-Japan Friendship Hospital, \#2 Yinghuayuan East Street, Chaoyang District, Beijing 100029, China. ${ }^{2}$ Department of Health Reform and Development, China-Japan Friendship Hospital, Beijing, China.

Received: 24 September 2017 Accepted: 18 April 2018

Published online: 23 April 2018

\section{References}

1. Wingerchuk DM, Hogancamp WF, O'Brien PC, Weinshenker BG. The clinical course of neuromyelitis optica (Devic's syndrome). Neurology. 1999;53(5):1107-14.

2. Wingerchuk DM, Pittock SJ, Lucchinetti CF, Lennon VA, Weinshenker BG. A secondary progressive clinical course is uncommon in neuromyelitis optica. Neurology. 2007;68(8):603-5.

3. Mealy MA, Wingerchuk DM, Palace J, Greenberg BM, Levy M. Comparison of relapse and treatment failure rates among patients with neuromyelitis optica: multicenter study of treatment efficacy. JAMA Neurol. 2014;71(3):324-30.

4. Jacob A, Matiello M, Weinshenker BG, Wingerchuk DM, Lucchinetti C, Shuster

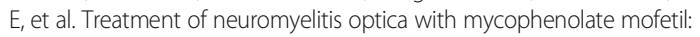
retrospective analysis of 24 patients. Arch Neurol. 2009;66(9):1128-33.

5. Huh SY, Kim SH, Hyun JW, Joung AR, Park MS, Kim BJ, et al. Mycophenolate mofetil in the treatment of neuromyelitis optica spectrum disorder. JAMA Neurol. 2014;71(11):1372-8.

6. Chen $H$, Zhang Y, Shi Z, Feng H, Yao S, Xie J, et al. The efficacy and tolerability of mycophenolate Mofetil in treating Neuromyelitis Optica and Neuromyelitis Optica Spectrum disorder in western China. Clin Neuropharmacol. 2016;39(2):81-7.

7. Xu Y, Wang Q, Ren HT, Qiao L, Zhang Y, Fei YY, et al. Comparison of efficacy and tolerability of azathioprine, mycophenolate mofetil, and cyclophosphamide among patients with neuromyelitis optica spectrum disorder: a prospective cohort study. J Neurol Sci. 2016 Nov 15:370:224-8.

8. Chen H, Qiu W, Zhang Q, Wang J, Shi Z, Liu J, et al. Comparisons of the efficacy and tolerability of mycophenolate mofetil and azathioprine as treatments for neuromyelitis optica and neuromyelitis optica spectrum disorder. Eur J Neurol. 2017:24(1):219-26.

9. Jeong $\mathrm{HH}$, Park B, Kim SH, Hyun JW, Joo J, Kim HJ. Comparative analysis of treatment outcomes in patients with neuromyelitis optica spectrum disorder using multifaceted endpoints. Mult Scler J. 2016;22(3):329-39.

10. Montcuquet A, Collongues N, Papeix C, Zephir H, Audoin B, Laplaud D, et al. NOMADMUS study group and the Observatoire Français de la Sclérose en plaques (OFSEP). Effectiveness of mycophenolate mofetil as first-line therapy in AQP4-lgG, MOG-lgG, and seronegative neuromyelitis optica spectrum disorders. Mult Scler. 2017;23(10):1377-84.

11. Wingerchuk DM, Lennon VA, Pittock SJ, Lucchinetti CF, Weinshenker BG. Revised diagnostic criteria for neuromyelitis optica. Neurology. 2006;66:1485-9.

12. Wingerchuk DM, Lennon VA, Lucchinetti CF, Pittock SJ, Weinshenker BG. The spectrum of neuromyelitis optica. Lancet Neurol. 2007;6(9):805-15.

13. Tackley G, O'Brien F, Rocha J, Woodhall M, Waters P, Chandratre S, et al. Neuromyelitis optica relapses: race and rate, immunosuppression and impairment. Mult Scler Relat Disord. 2016;7:21-5.

14. US Food and Drug Administration. Communication about an ongoing safety review of CellCept (mycophenolate mofetil) and Myfortic (mycophenolic acid). Rockville, MD: US Food and Drug Administration; 2015.

15. Fialho SC, Bergamaschi S, Neves FS, Zimmermann AF, Castro GR, Pereira IA Mycophenolate mofetil in primary Sjögren's syndrome: a treatment option for agranulocytosis. Rev Bras Reumatol. 2012;52(2):297-9.

16. Alexander S, Fleming DH, Mathew BS, Varughese S, Jeyaseelan V, Tamilarasi $V$, et al. Pharmacokinetics of concentration-controlled mycophenolate mofetil in proliferative lupus nephritis: an observational cohort study. Ther Drug Monit. 2014;36(4):423-32.

17. Lourdudoss C, Vollenhoven RV. Mycophenolate mofetil in the treatment of SLE and systemic vasculitis: experience at a single university center. Lupus. 2014;23(3):299-304.

\section{Ready to submit your research? Choose BMC and benefit from:}

- fast, convenient online submission

- thorough peer review by experienced researchers in your field

- rapid publication on acceptance

- support for research data, including large and complex data types

- gold Open Access which fosters wider collaboration and increased citations

- maximum visibility for your research: over $100 \mathrm{M}$ website views per year

At BMC, research is always in progress.

Learn more biomedcentral.com/submissions 